\title{
Avaliação de Eficácia do Programa de Controle da Doença de Chagas: Aspectos Metodológicos
}

\author{
Evaluation of the Efficacy of the Chagas' Disease Control Program: \\ Methodological Aspects
}

Mariângela Carneiro ${ }^{1}$

Carlos M. F. Antunes ${ }^{1}$

\begin{abstract}
CARNEIRO, M. E ANTUNES, C. M. F. Evaluation of the Efficacy of the Chagas'Disease Control Program: Methodological Aspects. Cad. Saúde Públ., Rio de Faneiro, 10 (supplement 2): 261-272, 1994.

The efficacy of the Chagas' Disease Control Program was estimated in Minas Gerais State. Areas with different intervention times (10 and 5 years) and without intervention (control) were compared in order to verify a possible reduction in Trypanosoma cruzi infection incidence rates among those born after the Control Program. The Program efficacy was estimated comparing this study's infection rates with rates published by the Chagas' Disease Serological Survey, conducted between 1975-1980. The cross-sectional comparison for the 2-6-year age group showed a reduction of $94.7 \%, 63.2 \%$, and $65.2 \%$ respectively, in areas where interventions had been carried for 10 years, 5 years, and in control areas. Cohort comparison, however indicated that reduction (52.6) occurred only in the area with 10 years of intervention. The data routinely collected by the Control Program were also analyzed and correlated with the incidence of $\underline{T}$. cruzi infection. The results showed association between the Control Program and reduction in $\underline{T}$. cruzi infection, measured by serological tests.
\end{abstract}

Key words: Chagas'Disease; Trypanosoma cruzi infection; Vector Control; Triatomine Vectors; Vectors Ecology; Program Efficay

\section{INTRODUÇÃO}

O Programa de Controle da Doença de Chagas (PCDCh), desenvolvido pelo Ministério da Saúde (MS) e pela Fundação Nacional de Saúde (FNS), é considerado hoje um dos maiores programas de controle de endemias no Brasil. A intervenção adotada, desde sua fase inicial, consistiu no controle regular dos hospedeiros intermediários (triatomíneos) domiciliados do Trypanosoma cruzi, agente etiológico da doença de Chagas, por meio da utilização de inseticidas de poder residual. Suas bases técnicas foram definidas na década de 1940 (Dias, 1946; Dias \& Pelegrino, 1948; Dias et al.,

\footnotetext{
${ }^{1}$ Departamento de Parasitologia do Instituto de Ciências Biológicas da Universidade Federal de Minas Gerais. Avenida Antônio Carlos, 6.627, Caixa Postal 486, Belo Horizonte, MG, 31270-901, Brasil.
}

1952); entretanto, suas ações ficaram limitadas, durante os primeiros 20 anos, a experimentos de campo isolados. Somente a partir de 1975, o PCDCh começou a ser estruturado como um programa de controle, e sua metodologia foi sendo adequada e normatizada de acordo com as necessidades decorrentes da própria evolução do trabalho (MS/Sucam, 1980; Silveira, 1985). A partir de 1983, por decisão política e com recursos financeiros adequados, o PCDCh atingiu, de forma sistemática, praticamente toda área endêmica do país (Lima, 1983).

As ações do PCDCh, podem ser esquematicamente apresentadas em três fases: (1) preparatória - com as atividades simultâneas de reconhecimento geográfico e levantamento de triatomíneos; (2) de ataque - com as atividades de borrifação de inseticidas e pesquisa anual de tritomíneos nos domicílios; e (3) de vigilância - com participação comunitária, 
consistindo no atendimento à notificação, feita pela comunidade, da presença de triatomíneos nos domicílios. Nas três fases, a pesquisa de triatomíneos e a borrifação de inseticidas foi realizada de acordo com a metodologia estabelecida para o programa (MS/Sucam, 1980).

A avaliação dos objetivos alcançados pelo PCDCh nas áreas trabalhadas é realizada, rotineiramente, pela análise de indicadores entomológicos. A fase de vigilância ou de consolidação do PCDCh só é instalada nos municípios quando são alcançados os seguintes resultados: ausência das espécies domiciliares Triatoma infestans e Triatoma rubrofasciata, ausência de espécies não nativas na área e ausência de colônias intradomiciliares de qualquer espécie de triatomíneo (MS/Sucam, 1986; Moreno et al., 1992). O PCDCh tem sido efetivo em controlar as espécies domiciliares de triatomíneos. O T. infestans, principal vetor do T. cruzi, está praticamente erradicado do país, sendo hoje encontrado somente em focos residuais. Em regiões onde persistem vetores nativos da área fora do ambiente domiciliar, os resultados, embora positivos, têm sido mais lentos (Dias, 1987). Por exemplo, as espécies atualmente encontradas com ampla distribuição no Estado de Minas Gerais são o T. sordida e o Panstrongylus megistus, capturados em números significativos no peridomicílio (Dias et al., 1985; MS/FNS, 1993). A maior dificuldade no controle dessas espécies está provavelmente relacionada à degradação rápida dos inseticidas expostos às condições ambientais e à dificuldade de captura e borrifação no peridomicílio (Oliveira-Filho, 1984; Diotaiuti, 1991).

Embora a avaliação entomológica tenha indicado a eficácia do PCDCh na redução da população de triatomíneos domiciliados, é recomendada a inclusão, em sua fase de planejamento, de avaliação sorológica (conduzida nas faixas etárias mais jovens) e clínica da população que vive em áreas sob intervenção. Esses componentes são importantes para determinar o impacto alcançado pelo programa nos indicadores de saúde da população, medir a duração dos efeitos das ações de controle e planejar medidas específicas, quando necessárias. Apesar do valor que se reserva à avaliação entomoló- gica, a proposta final do controle é a redução da transmissão (WHO, 1991).

Estudos realizados em algumas regiões do Brasil em áreas sob intervenção do PCDCh têm demonstrado que houve redução da infecção pelo T. cruzi na população humana nas áreas trabalhadas. Alguns autores inferem que essa redução está diretamente relacionada às ações de controle; outros, que a redução observada é conseqüência maior de mudanças sociais, econômicas e culturais ocorridas durante o período de intervenção (Caldas Jr., 1980; Silva, 1981; Buralli, 1985; Rocha e Silva et al., 1979; Souza et al., 1984). Esses estudos, por não incluírem grupos ou áreas de comparação (sem intervenção) ou não utilizarem metodologia adequada à avaliação de impacto, não identificam nem quantificam a redução da infecção humana que poderia ser atribuída somente às ações do programa.

A avaliação de resultados de programas de saúde pública procura determinar se ocorreram mudanças nos indicadores de saúde da população e, caso tenham ocorrido, se elas podem ser atribuídas ao programa que está sendo avaliado. A questão principal a ser respondida é definir se o objetivo desejado e alcançado pode ser conseqüência de um programa ou intervenção específica (Ibrahim, 1983; Holland, 1984). A principal dificuldade em avaliação de resultados é a de isolar o efeito alcançado pelo programa de outros fatores externos ou de confusão. As variáveis de confusão podem interferir na relação programa-resultados obtidos, causando associação indireta, super ou subestimando o resultado obtido (Kleinbaum et al., 1982; Rothman, 1986). Essa etapa de avaliação é muitas vezes de difícil realização, pois demanda longo período de observação para que seja mensurado o impacto das medidas no nível de saúde da população, além de requerer desenhos mais complexos de pesquisa (Ibrahim, 1983; Holland, 1984; Orman, 1990).

A avaliação de resultados de programas na saúde da população é considerada área de atuação da epidemiologia que, por meio da aplicação dos estudos epidemiológicos, permite inferir relações causais, definir eficácia e/ou efetividade e ter controle sobre o maior número 
possível de fatores externos ou de confusão (Ibrahim, 83; Holland, 1986; Orman, 1990).

A eficácia do PCDCh, em Minas Gerais, foi estimada, pela comparação de taxas de infecção pelo T. cruzi em áreas com e sem intervenção, na população nascida após as ações das medidas de controle. A hipótese operacional elaborada foi: a provável redução nas taxas de infecção pelo T. cruzi em áreas com intervenção, quando comparadas a áreas sem-intervenção, pode ser atribuída às ações do PCDCh?

$\mathrm{O}$ estudo epidemiológico utilizado nesta investigação foi o delineamento quase-experimental. Esse delineamento é conceituado como estudo epidemiológico em que o fator de interesse é controlado artificialmente. Os grupos de comparação não são formados por alocação aleatória, sendo essa a principal diferença com relação ao estudo experimental (Campbel \& Stanley, 1966; Kleinbaum et al., 1982; Susser, 1985; Briscoe et al., 1986; Orman, 1990; Carneiro, 1992; Carneiro \& Antunes, s/d). Na definição das áreas para implantação do PCDCh, realizada pela FNS em Minas Gerais, foram consideradas as facilidades operacionais e administrativas e contigüidade das áreas a serem trabalhadas. Taxas de infestação domiciliar por triatomíneos e prevalência da infecção pelo T. cruzi não foram determinantes nessa escolha.

\section{MATERIAL E MÉTODOS}

\section{Seleção da Área}

Para seleção das áreas investigadas, foram estabelecidos os seguintes critérios: (1) tempos diferentes de intervenções; (2) pertencimento à mesma região geográfica; (3) presença de $T$. infestans no primeiro levantamento de triatomíneos realizado no PCDCh; este último critério foi estabelecido por ser o T. infestans a espécie domiciliar mais importante em Minas Gerais. O município sem intervenção foi identificado como o único da região em que o PCDCh estava na fase de reconhecimento geográfico e levantamento de triatomíneos. Para a seleção dos municípios com intervenção, procurou-se excluir aqueles que não satisfizessem os critérios acima relacionados e, entre os restantes, escolher os que apresentassem características sociais, econômicas e culturais as mais semelhantes possíveis às do município sem intervenção. Entre aqueles semelhantes, a escolha foi determinada pelos municípios que ofereciam mais facilidades operacionais para o trabalho que seria conduzido no campo. As áreas selecionadas situam-se no norte e noroeste de Minas Gerais e são os municípios de Rubelita (Sem Intervenção), sem intervenção do PCDC; Montalvânia (Intervenção-5), com cinco anos de intervenção; e Capitão Enéas (Intervenção-10), com 10 anos de intervenção.

\section{Processo de Amostragem}

A investigação foi conduzida na região rural das três áreas trabalhadas, e a unidade de estudo foi o domicílio. O cálculo do tamanho da amostra necessária para o estudo baseou-se na definição dos parâmetros: (1) proporção de positivos da infecção para o T. cruzi encontrada no Inquérito Sorológico Nacional (ISN), realizado no país no período de 1976-1980 (Camargo et al., 1984), para a faixa etária de 2-14 anos em cada município; e (2) erro $\alpha=0,05$ (nível de significância) e $1-\beta=0,90$ (poder do teste). Determinou-se como suficiente a amostra de 400 crianças na faixa etária 2-14 anos por município, para detectar a redução de $50 \%$ na incidência de infecção pelo T. cruzi, comparando áreas com e sem intervenção (Snedecor \& Cochran, 1989).

Para o sorteio aleatório dos domicílios a serem investigados, foram utilizados os dados arquivados na Seção de Estatística da MS/FNS$M G$, referentes à fase de Reconhecimento Geográfico (RG), efetuado no início do programa em cada município e atualizado em fases posteriores. O sorteio ocorreu em duas etapas, amostragem aleatória simples para seleção das localidades e amostragem aleatória proporcional para seleção das unidades domiciliares. Foram estabelecidos os seguintes critérios de estratificação: trabalhar todos os domicílios, nas localidades com menos de 10 casas; nas localidades que apresentassem entre 11 e 30 domicílios, 
trabalhar metade dos domicílios; nas localidades que apresentassem entre 31 e 60 domicílios, trabalhar um terço dos domicílios; nas localidades com mais de 60 domicílios, trabalhar 10\% dos domicílios.

As casas foram sorteadas até completar o número de pessoas, segundo a amostra necessária. Após o sorteio, as casas a serem pesquisadas foram assinaladas no mapa correspondente a cada localidade.

\section{Dados Coletados e Testes Sorológicos}

De todos os participantes coletaram-se informações por questionário pré-codificado, desenhado especialmente para a investigação. As entrevistas foram realizadas por uma equipe de entrevistadores previamente treinados e de posse de um Manual de Orientação. Os dados incluíam, além de informações pessoais, as características da habitação (domicílio e peridomicílio), conhecimento sobre doença de Chagas e transmissores, relato dos participantes sobre presença de triatomíneos nas habitações, melhoria da habitação realizada pelos moradores no período da intervenção, e identificação de possíveis fatores ligados à infecção ou ao PCDCh. No momento da entrevista coletou-se também amostra de sangue de todos os participantes, para realização de testes sorológicos a fim de identificar infecção pelo T. cruzi. Os testes sorológicos foram realizados no Laboratório de Parasitologia da Fundação Ezequiel Dias, Minas Gerais. As reações foram processadas por Reação de Imunofluorescência Indireta e Reação de Hemaglutinação Indireta.

\section{Dados Secundários}

A fonte básica para coleta de dados secundários foi o modelo de captura de triatomíneos utilizado na rotina do PCDCh (CCDCh-10), que é preenchido pelas equipes de campo da FNS, durante as pesquisas de triatomíneos realizadas nas habitações. As informações foram coletadas visando à análise da série histórica e epidemiológica nos municípios, em face das medidas de controle. Esses modelos estavam arquivados na Seção de Estatística da MS/FNS-MG. Foram identificados nos três municípios todos os domicílios sorteados para o estudo e analisadas as informações coletadas nesses formulários de campo. Para os municípios Intervenção-10 e Intervenção-5, coletaram-se dados correspondentes a 10 e cinco anos de intervenção, respectivamente. Para o município Sem Intervenção, os dados coletados referem-se ao trabalho de levantamento triatomínico inicial. O modelo (CCDCh-10) para captura de triatomíneos, que serviu de fonte para as informações coletadas, só é preenchido quando a casa é positiva com relação à captura de triatomíneos. Partiu-se da premissa de que, quando não existia o modelo preenchido, a casa havia sido negativa nas capturas de triatomíneos realizadas durante as fases do PCDCh.

\section{Análise de Dados}

A análise realizada, utilizando os programas Statistical Package for the Social Science SPSS/PC, versão 3.0 (Norusis, 1988a e 1988b) e Epi-Info, versão 5.1b (Dean et al., 1990), constou das seguintes etapas: (1) análise comparativa da distribuição de freqüências para as variáveis coletadas pela entrevista (amostra total, menores de 10 anos e infectados e não infectados pelo T. cruzi); (2) pesquisa de possíveis variáveis de confusão; (3) análise dos dados secundários; e (4) análise de eficácia do PCDCh.

Com relação aos dados secundários, foram analisados: (1) local de captura dos triatomíneos (intradomicílio ou peridomicílio); (2) identificação da espécie de triatomíneo capturada por domicílio; e (3) correlação com os dados encontrados na investigação.

A eficácia do PCDCh foi estimada comparando os resultados obtidos nessa investigação com os resultados do Inquérito Sorológico Nacional, considerados informação basal em cada município. Foram feitas comparações seccionais e análise de coorte para os grupos etários de 2-6 e 7-14 anos.

\section{RESULTADOS}

O trabalho foi realizado em 470 domicílios rurais, e 2354 indivíduos fizeram parte da amostra; desses, 854 (36,3\%) eram menores de 
10 anos. Na primeira etapa da análise, a comparação entre as três áreas indicou diferenças estatísticas em alguns fatores, que foram então pesquisados como possíveis variáveis de confusão. Como nenhuma das váriaveis testadas foi caracterizada como variável de confusão, as diferenças encontradas na análise da eficácia nas áreas investigadas podem ser atribuídas ao PCDCh.

\section{Análise dos Dados Secundários Coletados pelo PCDCh nos Municípos Estudados}

Os índices de positividade por ciclo foram calculados considerando, no numerador, o número de casas positivas nas capturas realizadas pelo PCDCh e, no denominador, o número de casas trabalhadas na investigação. Esses índices foram calculados: (1) por unidade domiciliar positiva e (2) por capturas realizadas no intra e peridomicílio. Na primeira etapa, não foram consideradas as espécies de triatomíneos capturadas; na segunda etapa, foram consideradas as espécies mais importantes no ciclo epidemiológico.

Para o município Sem Intervenção, os índices calculados referem-se somente à fase inicial, levantamento triatomínico (LT). No total de 164 casas trabalhadas no estudo, $14,6 \%$ (24) foram positivas com captura de triatomíneos, $\mathrm{e}$, delas, $4 \%$ (7) foram positivas com captura no intradomicílio, e $11 \%$ (17) com captura no peridomicílio. Nas casas com captura no intradomicílio, a espécie capturada foi o $T$. infestans. No peridomicílio, foram capturadas as espécies T. infestans e T. sordida, sendo $1 \%$ (2) das casas com $T$. infestans e 9\% (15) com $T$. sordida.

No município Intervenção-5 foram trabalhadas 146 casas no total. Os resultados referentes à fase de levantamento triatomínico (LT), até a fase de avaliação VI (AV-VI), ou seja, cinco anos de programa, encontram-se no Gráfico 1. As espécies assinaladas foram o $T$. infestans e T. sordida, em todas as fases do programa.

Para o município Intervenção-10, foram trabalhadas 160 casas. Os resultados referentes à fase levantamento triatomínico (LT) até avaliação XVI (AV-XVI), ou seja, 10 anos de programa, encontram-se no Gráfico 2. As espécies assinaladas foram o T. infestans, presente até a AV-VIII e o T. sordida, encontrado em praticamente todas as avaliações. Nas avaliações AV-XII e AV-XVI, foram encontradas Panstrongylus geniculatus e T. vitticeps, sem significado epidemiológico, pois foram capturados somente em uma casa em cada uma das avaliações.

\section{Análise da Eficácia do PCDCH}

\section{Comparação Seccional ou Transversal}

A Tabela 1 apresenta as taxas de prevalência por município comparadas aos resultados do Inquérito Sorológico Nacional (ISN). Os dados indicam redução na prevalência da infecção nos municípios Sem Intervenção e Intervenção-5 e aumento no município Intervenção-10. Essas diferenças mantiveram-se após ajustamento por idade.

Para a comparação por faixa etária, utilizouse a mesma divisão de classes apresentada no ISN (Tabela 2). Os dados são apresentados como distribuição proporcional de participantes infectados em cada faixa etária. A comparação transversal mostrou queda na proporção de positivos nas faixas etárias mais baixas (2-6 e 7-14 anos) nas três áreas, à exceção do município Intervenção-5, na faixa etária 7-14 anos. Essas diferenças, em valores relativos para a faixa etária 2-6 anos, são apresentada na Tabela 3; a maior redução $(94,7 \%)$ foi observada no município Intervenção-10.

\section{Comparação da Coorte Etária}

A análise, para as faixa etárias mais jovens, mostrou proporções estáveis de infecção na coorte de 7-14 anos nas áreas trabalhadas. Para a coorte de 2-6 anos, foram observados aumento significativo nos municípios Sem Intervenção e Intervenção-5 e redução no município Intervenção-10 (Tabela 2). Essas diferenças, em valores relativos, podem ser observadas na Tabela 4. 
FIGURA 1. Eficácia do PCDCh-MG na Área de Intervenção-5
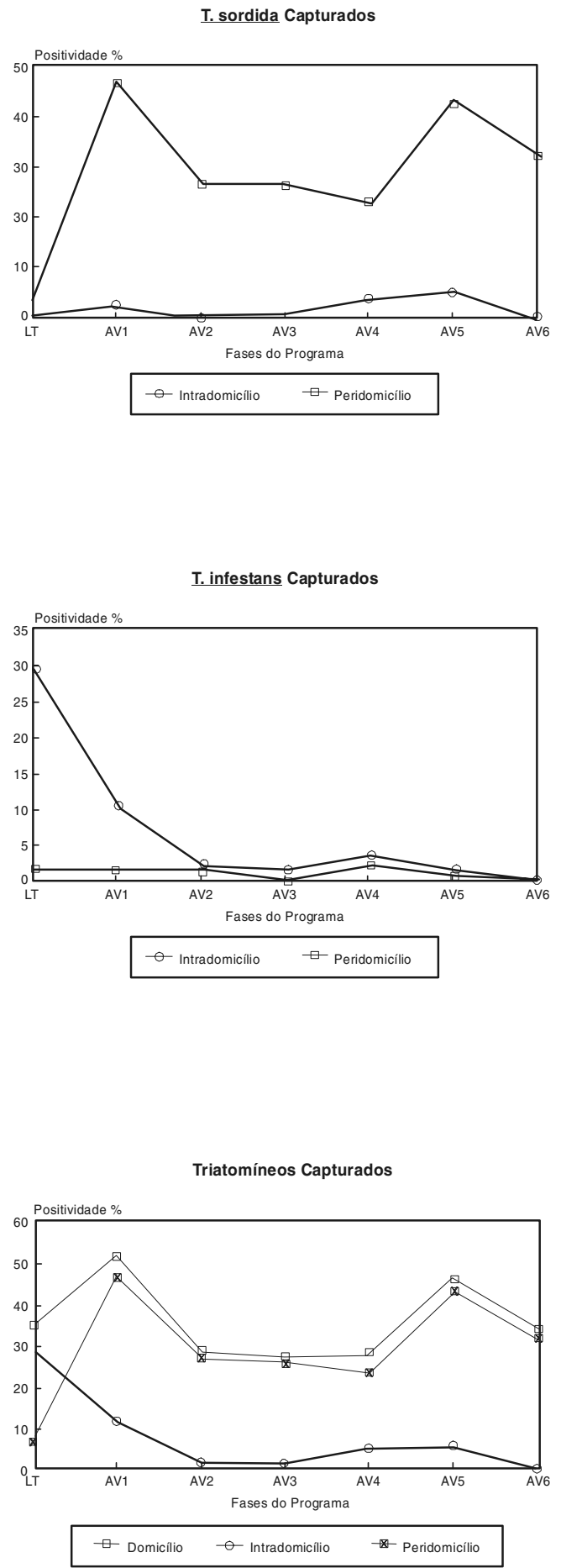

Fonte: MS/FNS-MG - Seção de Estatística. 
FIGURA 2. Eficácia do PCDCh-MG na Área de Intervenção-10

T. sordida Capturados

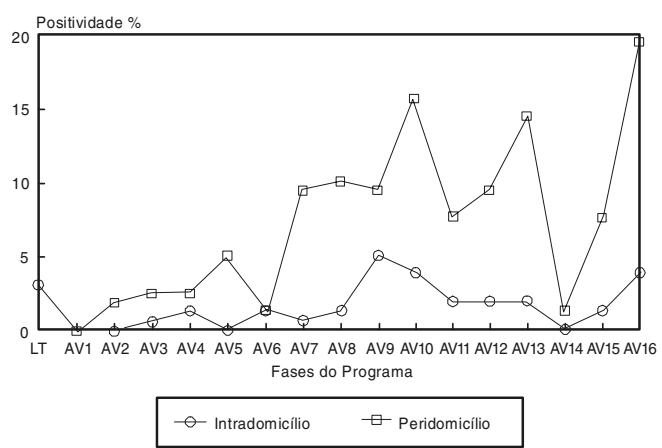

T. Infestans Capturados

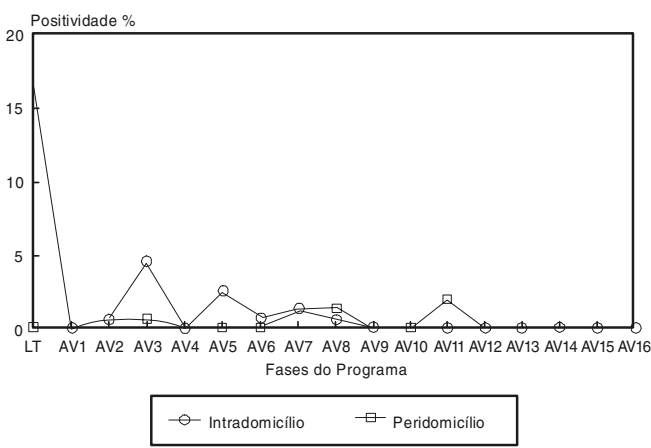

Triatomíneos Capturados

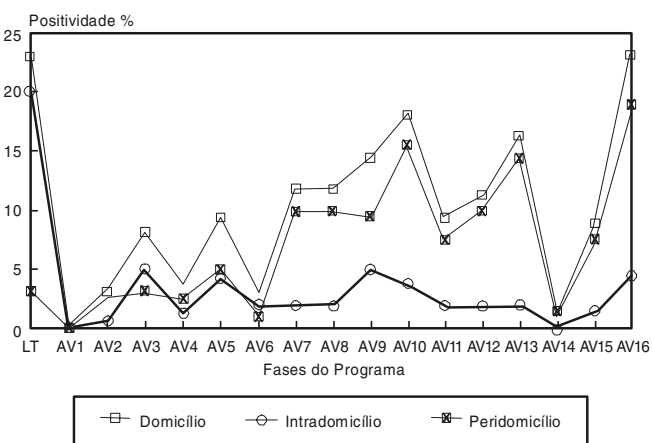

Fonte: MS/FNS-MG - Seção de Estatística. 
TABELA 1. Comparação dos Índices de Infecção pelo T. cruzi nas Áreas Investigadas Minas Gerais, 1987

\begin{tabular}{lcccc}
\hline \hline & \multirow{2}{*}{$\begin{array}{c}\text { Inquérito } \\
\text { Nacional }\end{array}$} & \multicolumn{3}{c}{${\text { Investigação }(\%)^{2}}^{1}$} \\
\cline { 3 - 5 } Anveas & $(\%)$ & Total & $>5 \operatorname{anos}^{3}$ & $>10 \operatorname{anos}^{4}$ \\
\hline Sem Intervenção & 31,1 & 12,8 & & \\
Intervenção-5 & 13,9 & 7,8 & 7,7 & 20,7 \\
Intervenção-10 & 10,2 & 14,1 & & 2 \\
\hline \hline
\end{tabular}

${ }^{1}$ Inquérito Sorológico Nacional (1975-1980)

${ }^{3}$ Excluídos menores de 5 anos

${ }^{2}$ Avaliação do Programa

${ }^{4}$ Excluídos menores de 10 anos

TABELA 2. Comparação dos Índices de Infecção pelo T. cruzi nas Áreas Investigadas por Faixa Etária Minas Gerais, 1987

\begin{tabular}{|c|c|c|c|c|c|c|}
\hline \multirow[b]{3}{*}{$\begin{array}{l}\text { Faixa Etária } \\
\text { (anos) }\end{array}$} & & & \multicolumn{4}{|c|}{ Áreas Com Intervenção } \\
\hline & \multicolumn{2}{|c|}{ Sem Intervenção } & \multicolumn{2}{|c|}{5 Anos } & \multicolumn{2}{|c|}{10 Anos } \\
\hline & $\mathrm{ISN}^{1}$ & $\mathrm{INV}^{2}$ & ISN & INV & ISN & INV \\
\hline $2-6$ & $\underline{6,9}$ & 2,4 & $\underline{15,5}$ & 5,7 & $\underline{19,0}$ & 1,0 \\
\hline $7-14$ & $\underline{37,5}$ & 13,1 & $\underline{26,6}$ & $\mathbf{5 4 , 7}$ & $\underline{33,0}$ & $\mathbf{9 , 0}$ \\
\hline $15-29$ & 19,0 & 36,9 & 20,0 & $\underline{22,6}$ & 4,7 & 34,0 \\
\hline $30-44$ & 18,0 & 25,0 & 20,0 & 11,3 & 28,5 & 17,0 \\
\hline+45 & 18,0 & 22,6 & 17,7 & 5,7 & 19,2 & 39,0 \\
\hline
\end{tabular}

${ }^{1}$ Inquérito Sorológico Nacional (1975-1980) $\quad$ 2 Avaliação do Programa

TABELA 3. Comparação dos Índices de Infecção pelo T. cruzi nas Áreas Investigadas: Redução Relativa no mesmo Grupo Etário, em Diferentes Épocas Minas Gerais, 1987

\begin{tabular}{|c|c|c|c|}
\hline Áreas & Grupo Etário & $\%$ Positivos & $\sigma(\%)$ \\
\hline \multicolumn{4}{|l|}{ Sem Intervenção } \\
\hline - Inquérito Nacional ${ }^{1}$ & $2-6$ & 6,9 & $65,2 \downarrow$ \\
\hline - Avaliação do Programa ${ }^{2}$ & $2-6$ & 2,4 & \\
\hline \multicolumn{4}{|l|}{ Intervenção-5 } \\
\hline • Inquérito Nacional ${ }^{1}$ & $2-6$ & 15,5 & $63,2 \downarrow$ \\
\hline - Avaliação do Programa ${ }^{2}$ & $2-6$ & 5,7 & \\
\hline \multicolumn{4}{|l|}{ Intervenção-10 } \\
\hline - Inquérito Nacional ${ }^{1}$ & $2-6$ & 19,0 & $94,7 \downarrow$ \\
\hline - Avaliação do Programa ${ }^{2}$ & $2-6$ & 1,0 & \\
\hline
\end{tabular}

${ }^{1} 1975-80 \quad 21987$ 
TABELA 4. Comparação dos Índices de Infecção pelo T. cruzi nas Áreas Investigadas: Redução Relativa na Coorte Etária de 2 - 6 anos Minas Gerais, 1987

\begin{tabular}{lccc}
\hline \hline Áreas & Grupo Etário & $\%$ Positivos & $\sigma(\%)$ \\
\hline Sem Intervenção & $2-6$ & 6,9 & $89,9 \uparrow$ \\
• Inquérito Nacional $^{1}$ & $7-14$ & 13,1 & \\
• Avaliação do Programa $^{2}$ & & & \\
Intervenção-5 $^{\text {• Inquérito Nacional }}{ }^{1}$ & $2-6$ & 15,5 & $248,4 \uparrow$ \\
• Avaliaçãa do Programa $^{2}$ & $7-14$ & 54,4 & \\
Intervenção-10 $^{\text {• Inquérito Nacional }}{ }^{1}$ & & & \\
• Avaliação do Programa $^{2}$ & $2-6$ & 19,0 & $52,6 \downarrow$ \\
\hline \hline
\end{tabular}

${ }^{1} 1975-80 \quad{ }^{2} 1987$

\section{DISCUSSÃO}

Os resultados encontrados mostraram associação entre as ações do PCDCh e a redução da infecção pelo T. cruzi, estimada por testes sorológicos.

A comparação seccional das estimativas de prevalência dos resultados da investigação com os obtidos no ISN mostrou redução da infecção somente nas áreas Sem Intervenção e Intervenção-5; esses resultados mantiveram-se após remoção, da análise, daqueles nascidos após a intervenção do programa (Tabela 1). Esses resultados, aparentemente contraditórios, são devidos ao emprego da análise de curvas seccionais em áreas onde foi observada acentuada redução na transmissão durante o período de tempo estudado. Nessas situações, a análise de coortes etárias deve ser empregada (Frost, 1939; Lilienfeld \& Lilienfeld, 1980; Mausner \& Kramer, 1985; Susser, 1987). Entretanto, deve ser ressaltado que o aumento observado na prevalência da infecção na área Intervenção-10, quando retirados os menores de 10 anos, fornece evidência indireta da eficácia do PCDCh.

A análise seccional por faixa etária (Tabela 2) evidenciou redução da infecção no grupo etário 2-6 anos, nas três áreas; entretanto, a maior redução foi observada na área Intervenção-10. No grupo etário 7-14 anos, houve redução nas áreas Sem Intervenção e Intervenção-10, e aumento na área Intervenção-5. Esses resultados podem ser atribuídos à inclusão, nesse grupo, de participantes nascidos antes da implementação do PCDCh. A divisão por essas faixas etárias foi necessária para que os dados pudessem ser comparados com os obtidos pelo ISN; entretanto, esse foi um fator limitante para comparações mais precisas. A quantificação da redução na faixa etária 2-6 anos (Tabela 3) sugere queda da infecção nas três áreas; esse resultado, provavelmente um artefato, pode ser atribuído à limitação da análise seccional.

A análise de coorte etária, mais adequada para estimar a eficácia do PCDCh, acompanhou os diversos grupos, considerando sua experiência comum frente à infecção pelo T. cruzi em um período de tempo definido. Os resultados obtidos mostraram redução, para a coorte de 2-6 anos, somente na área Intervenção-10. O acompanhamento da coorte de 7-14 anos mostrou que os resultados obtidos não diferiram dos obtidos no ISN, em nenhum dos municípios trabalhados (Tabelas 2 e 4). Esse achado reforça a conclusão de que a redução observada para a faixa etária 2-6 anos é devida às ações do PCDCh.

Para o município Intervenção-10, os resultados encontrados na análise da coorte etária de 2-6 anos mostram queda no índice de infeção pelo T. cruzi, quando se esperavam valores semelhantes. As explicações prováveis seriam: (1) as faixas etárias adotadas pelo ISN (2-6 e 7-14 anos) não são as mais adequadas para 
análise; (2) maior taxa de mortalidade ocorrida entre as crianças infectadas pelo $T$. cruzi no período de tempo decorrido entre a realização do ISN e a investigação; e (3) a migração, que pode ter ocorrido durante esse mesmo período de tempo.

No município Intervenção-5, houve aumento muito acentuado na taxa de infecção pelo $T$. cruzi, no período de 10 anos decorrido entre o ISN e esta investigação, na coorte etária de 2-6 anos. As possíveis explicações que poderiam justificar esse aumento acentuado são: (1) a divisão da classe etária incluindo crianças nascidas antes da intervenção, expostas à transmissão vetorial; (2) o tempo decorrido entre a implementação do programa e sua avaliação, insuficiente para determinação da eficácia; esse fato pode estar associado ao grande número de triatomíneos existentes nesse município no levantamento de triatomíneos inicial.

Os resultados do ISN, embora publicados como taxa de prevalência por município, estão disponíveis para os diversos grupos etários em distribuição proporcional. Essas proporções podem ser comparadas, mas não podem ser utilizadas como estimativas de incidência ou risco.

Os resultados obtidos nesta investigação foram confirmados pela análise dos dados do PCDCh quanto à captura de triatomíneos encontrados nos dois municípios com intervenção. Foi demonstrado, nessas áreas, o sucesso do controle do T. infestans, espécie domiciliada. A curva que representa a positividade de casas com captura dessa espécie no intradomicílio é drasticamente descendente nas duas áreas. No município Intervenção-10, a queda é mais brusca já no início do PCDCh; no município Intervenção-5, ocorre a partir do segundo ano do programa. A redução da população domiciliada de $T$. infestans, foi também comprovada no Estado de São Paulo (Rocha e Silva, 1979; Souza et al., 1984) e em todas as áreas endêmicas do Brasil (Dias, 1987). A outra espécie capturada na região é o $T$. sordida, que mostrou, a partir do início do programa, aumento da população nos dois municípios trabalhados; foi capturada principalmente no peridomicílio. No município Intervenção-10, ao longo da série histórica analisada, em nenhum momento apresentou densidades importantes no intradomicílio.
Mesmo considerando as mudanças sociais, econômicas e culturais que estão ocorrendo no Estado de Minas Gerais, esta investigação comparou áreas semelhantes quanto a essas variáveis, retirando efeitos externos ao PCDCh, que poderiam reduzir a incidência da infecção pelo T. cruzi. As áreas foram comparadas como se fossem pareadas por esses fatores.

O estudo epidemiológico quase experimental mostrou-se adequado para avaliação do PCDCh por ser indicado, principalmente, quando: (1) o método aleatório para escolha das áreas a serem ou não trabalhadas não for utilizado; (2) os programas atingirem grandes populações; (3) a avaliação for implementada concomitantemente à execução das ações do programa; e (4) por questões éticas, uma vez que o programa não precisa ser interrompido para ser avaliado (Kleinbaum et al., 1982; Briscoe et al., 1986; Orman, 1990). Na escolha desse delineamento, foram considerados: (1) que o PCDCh se encontrava com metodologia definida há vários anos; (2) que sua efetividade era comprovada no controle de triatomíneos domiciliados; e (3) que na fase de planejamento da investigação, toda área endêmica do Estado de Minas Gerais já se encontrava sob intervenção ou com planejamento pronto para que se iniciasse o PCDCh.

\section{RESUMO}

CARNEIRO, M. \& ANTUNES, C. M. F. Avaliação de Eficácia do Programa de Controle da Doença de Chagas: Aspectos Metodológicos. Cad. Saúde Públ., Rio de Janeiro, 10 (suplemento 2): 261-272, 1994.

A eficácia do Programa de Controle da Doença de Chagas (PCDCh) foi estimada em um estudo conduzido no Estado de Minas Gerais. Áreas com diferentes tempos de intervenção (cinco e 10 anos) e sem intervenção (controle) foram comparadas para verificar possível redução na incidência da infecção pelo Trypanosoma cruzi nos habitantes nascidos após a implantação do PCDCh. A eficácia do PCDCh foi estimada pela comparação dos resultados da infecção pelo T. cruzi, com resultados obtidos pelo Inquérito Sorológico Nacional (1975-1980), realizado antes do início do Programa. A 
comparação seccional para a faixa etária de 2-6 anos indicou redução nos índices de infecção de 94,7\% (área de 10 anos), 63,2\% (área de cinco anos) e 65,2\% (área sem intervenção). Entretanto, a análise de coorte, para as faixa etárias de 2-6 anos e 7-14 anos, mostrou que a redução observada $(52,6 \%)$ ocorreu somente na área com 10 anos de intervenção. A análise dos dados sobre triatomíneos coletados na rotina do PCDCh estabeleceu correlação com a redução de infecção pelo T. cruzi, observada na área com intervenção. Os resultados encontrados mostraram associação entre as ações do PCDCh e a redução da infecção pelo T. cruzi, estimada por testes sorológicos.

Palavras-Chave: Doença de Chagas; Trypanosoma cruzi; Controle de Vetores; Triatomíneos; Ecologia de Vetores; Eficácia de Programas

\section{REFERÊNCIAS BIBLIOGRÁFICAS}

BRISCOE, J.; FEACHEM, R. G. \& RAHAMAN, $M$. M., 1986. Evaluation Health Impact. Water Supply, Sanation, and Hygiene Education. Ottawa: IDRC.

BURALLI, G. M., 1985. Estudo do Controle de Triatomíneos Domiciliados no Estado de São Paulo. Tese de Mestrado, São Paulo: Universidade de São Paulo.

CALDAS Jr., A. L., 1980. Epidemiologia e Controle da Doença de Chagas. Relação com Estrutura Agrária na Região de Sorocaba, SP. Tese de Mestrado, São Paulo: Universidade de São Paulo.

CAMARGO, M. E.; SILVA, G. R.; CASTILHO, E. A. \& SILVEIRA, A. C., 1984. Inquérito sorológico da prevalência da infecção chagásica no Brasil - 1975/1980. Revista do Instituto de Medicina Tropical de São Paulo, 26: 192-204.

CAMPBEL, D. T. \& STANLEY, J., 1966. Experimental and Quasi-Experimental Designs for Research. Boston: Honghton Mifflin Company.

CARNEIRO, M., 1992. Avaliação de Eficácia de Programas de Saúde Pública: Estudo Epidemiológico Quase-Experimental. Eficácia do Programa de Controle da Doença de Chagas. Tese de Doutorado, Belo Horizonte: Universidade Federal de Minas Gerais.
CARNEIRO, M. \& ANTUNES, C. M. F., s/d. A quasi-experimental epidemiological model for evaluating public health programs: Efficacy of Chagas' disease control program in Brazil. Bulletin of the World Health Organization. (no prelo)

DEAN, A. G.; BURTON, A. H. \& DICKER, R. C., 1990. Epi-Info Version 5: A World Processing Database, and Statistics System for Epidemiology on Microcomputer. Atlanta, GA: USD Inc.

DIAS, E., 1946. Profilaxia da Doença de Chagas. Resumo das principais atividades do Centro de Estudos do Instituto Oswaldo Cruz em Bambuí, MG. Brasil Médico, 60: 161-163.

DIAS, E. \& PELLEGRINO, J., 1948. Alguns ensaios com gamaxane, no combate a transmissores da doença de Chagas. Brasil Médico, 62: 185-191.

DIAS, E.; PELLEGRINO, J. \& CASTRO, J. A., 1952. Ensaio experimental da luta contra triatomíneos por meio de inseticidas de ação residual. Revista Brasileira de Malariologia e Doenças Tropicais, 4: 22-46.

DIAS, J. C. P., 1987. Control of Chagas Disease in Brazil. Parasitology Today, 3): 336-341.

DIAS, J. C. P.; LOYOLA, C. C. P. \& BRENER, S., 1985. Doença de Chagas em Minas Gerais: situação atual e perspectivas. Revista Brasileira de Malariologia e Doenças Tropicais, 37: 07-28.

DIOTAIUTI, L., 1991. Importância Atual e Perspectiva de Controle do Triatoma sordida em Minas Gerais. Tese de Doutorado, Belo Horizonte: Universidade Federal de Minas Gerais.

FROST, W. H., 1939. The age selection of mortality from tuberculose in sucessive decades. American Fournal of Hygiene, 30: 91-96.

HOLLAND, W. W., 1984. Evaluation of Health Care. Oxford: Oxford University Press.

IBRAHIM, M. A., 1983. Epidemiology: application to health services. Fournal of Health Administration, 1: 327-369.

KLEINBAUM, D. G.; KUPPER, L. L. \& MORGENSTERN, H., 1982. Epidemiologic Research: Principles and Quantitative Methods. New York: VNB.

LILIENFELD, A. M. \& LILIENFELD, D. E., 1980. Foundations of Epidemiology. New York: Oxford University Press.

LIMA, J. T. F., 1983. Incremento do programa de controle da doença de Chagas no Brasil. Revista da Sociedade Brasileira de Medicina Tropical, 16: 128-129.

MAUSNER, J. \& KRAMER, S., 1985. Epidemiology - An Introductory Text. Philadelphia: W. B. Saunders Company. 
MS (Ministério da Saúde) \& SUCAM (Superintêndencia de Campanhas de Saúde Pública), 1980. Manual de Normas Técnicas da Campanha de Controle da Doença de Chagas. Brasília, DF: Centro de Documentação do Ministério da Saúde.

, 1986. I Seminário sobre Vigilância Epidemiológica em Doença de Chagas. Documento Interno. Brasília, DF: MS. (Mimeo.)

MS (Ministério da Saúde) \& FNS (Fundação Nacional de Saúde), 1993. Relatório Técnico do Programa de Controle da Doença de Chagas. Documento Interno. Brasília, DF: MS. (Mimeo.)

MORENO, E. C.; CASTRO, J. C. F.; AZEREDO, B. V. M.; CARNEIRO, M.; DIOTAIUTI, L. \& DIAS, J. C. P. 1992. Vigilância Epidemiológica no Programa de Controle da doença de Chagas em Minas Gerais. II Congresso Brasileiro de Epidemiologia. Belo Horizonte: Associação Brasileira de Pós-Graduação em Saúde Coletiva. (Mimeo.)

NORUSIS, M. J., 1988a. SPSS/PC + V2.0. Base Manual. Chicago: SPSS Inc. , 1988b. SPSS/PC + Advanced Statistcs V 2.0. Chicago: SPSS Inc.

OLIVEIRA-FILHO, A. M., 1984. New alternatives for Chagas disease control. Memórias do Instituto Oswaldo Cruz, 79: 117-123.

ORMAN, A. R., 1990. Investigación sobre sistemas de salud: métodos y escolhas. Foro Mundial de la Salud, 11: 288-294.

ROCHA E SILVA, E. O.; GUARITA, O. F. \& ISHIHATA, G. K., 1979. Doença de Chagas: atividades de controle de transmissão no Estado de São Paulo. Revista Brasileira de Malariologia e Doenças Tropicais, 31: 99-1193.
ROTHMAN, K. J., 1986. Modern Epidemiology. Boston: Little Braun and Company.

SILVA, L. J., 1981. Evolução da Doença de Chagas no Estado de São Paulo. Tese de Doutorado, Ribeirão Preto: Universidade de São Paulo.

SILVEIRA, A. C., 1985. O programa de controle da doença de Chagas no Brasil. Annales des Sociétés Belges de Medicine Tropicale, 65 (suppl. 1): 137-148.

SNEDECOR, GM. \& COCHRAN, W. G., 1989. Statistical Methods. Ames: Iowa State University Press/AMES.

SOUZA, A. G.; VALERIO-WANDERLEY, D. M.; BURALLI, G. M. \& ANDRADE, J. C. R., 1984. Consolidation of the control of Chagas disease vectors in the state of São Paulo. Memórias do Instituto Oswaldo Cruz, 79: 125-131.

SUSSER, M., 1985. Epidemiolgy in the United States after II World. The evolution of technique. Epidemiology Reviews, 7: 147-177.

, 1987. Period effects, generation effects and age effects in peptic ulcer mortality. In: Epidemiology, Health E Society. Selected Papers (M. Susser), pp. 140-150, New York: Oxford University Press.

WHO (World Health Organization), 1991. Control of Chagas Disease. Report of a WHO Exppert Committee. Geneva: WHO. (Techical Report Series, 811) 